CLINICAL STUDY

\title{
Comparable reduction of the visceral adipose tissue depot after a diet-induced weight loss with or without aerobic exercise in obese subjects: a 12-week randomized intervention study
}

Tore Christiansen, Søren K Paulsen, Jens M Bruun, Kristian Overgaard ${ }^{1}$, Steffen Ringgaard ${ }^{2}$, Steen B Pedersen, Vincenzo Positano ${ }^{3}$ and Bjørn Richelsen

Department of Medicine and Endocrinology C, Aarhus University Hospital, Aarhus Sygehus, Tage Hansensgade 2, DK-8000 Aarhus C, Denmark,

${ }^{1}$ Department of Sport Science, University of Aarhus, Aarhus, Denmark, ${ }^{2}$ MR-Research Centre, Aarhus University Hospital, Aarhus, Denmark and

${ }^{3}$ MRI Laboratory, G. Monasterio Foundation, Pisa, Italy

(Correspondence should be addressed to T Christiansen; Email: tore.christiansen@ki.au.dk)

\begin{abstract}
Objective: Weight loss with preferential effect on the visceral adipose tissue (VAT) depot could have important clinical benefits. In this study, we investigated the independent and combined effect of regular exercise and diet induced weight loss on body fat distribution.

Design: Randomized control design of i) exercise-only (EXO; 12 weeks of exercise without dietrestriction), ii) hypocaloric-diet (DIO; 8 weeks of very low energy diet (VLED $600 \mathrm{kcal} /$ day) followed by 4-weeks weight maintenance diet) and iii) hypocaloric-diet and exercise (DEX; 8 weeks VLED $800 \mathrm{kcal} /$ day $+\mathrm{a}$ 4-week weight maintenance diet combined with exercise throughout the 12 weeks). Subjects: Seventy-nine obese males and females were included.

Measurements: Body fat distribution was quantified by magnetic resonance imaging (MRI)-technology. Results: In the EXO group, the weight loss $(3.5 \mathrm{~kg})$ and the relative reduction in VAT (18\%) was significantly lower compared with the weight losses in the DIO and DEX groups $(12.3 \mathrm{~kg} ; P<0.01)$ and to the reduction in VAT $(30-37 \% ; P<0.01)$. In all the three groups, the relative reduction of VAT was higher as compared with the reduction in fat mass (FM; combining all fat depots determined by MRI; $P<0.01$ for all comparisons). The changes in VAT were associated with changes in FM and related to the initial VAT/FM ratio $\left(r^{2}=0.72 ; P<0.01\right)$.

Conclusion: Exercise has no additional effects in reduction of the VAT depot, compared with the major effects of hypocaloric diet alone. In addition, the effects of exercise per se on VAT are relatively limited. The effects on the VAT depot are closely associated with changes in total FM.
\end{abstract}

European Journal of Endocrinology 160 759-767

\section{Introduction}

Obesity, in particular excess visceral adipose tissue (VAT), is associated with the metabolic syndrome (1) resulting in increased morbidity and mortality (2). By contrast, accumulation of body fat in the subcutaneous gluteal-femoral adipose tissue (GFAT) is generally less associated with health problems or may even mediate some protection against cardiovascular diseases (3-5). These findings suggest that fat distribution and particularly the ratio between VAT and GFAT may be of importance for the obesity-related health complications. The cornerstone in the treatment of obesity is dietinduced weight loss, but it is well-known that weight loss is generally difficult to obtain and to maintain $(6,7)$. Since VAT seems to be the most important fat depot in relation to health complications, treatments that could have preferential effects in reducing VAT have attracted much interest. Both endogenous factors (e.g., genes and sex) and exogenous factors (e.g., diet, hormones (corticosteroids and some sex hormones)) and pharmacology such as peroxisome proliferator-activated receptor (PPAR) $\gamma$-agonists and some antiviral treatments (protease inhibitors) have been demonstrated to be able to affect fat distribution (8-10).

Investigations from several groups suggest that exercise may also favor a reduction in VAT with or without general weight loss (11-16). Regular exercise without diet restriction has been found to reduce the VAT depot with up to $48 \%$ (15) and improvement of $\mathrm{VO}_{2}$ max during exercise interventions has been associated with higher reduction in VAT (17). This possible specific effect on VAT may also explain some of the positive 'weight independent' effect of exercise on the metabolic syndrome (18). However, not all investigations have found this preferential effect of exercise on VAT $(19,20)$. One explanation for the observed differences could be due to the fact that VAT 
has been determined by different and sometimes less accurate methods.

With newer technologies such as magnetic resonance imaging (MRI) and computed tomography (CT) it is possible to accurately quantify the size of the various fat depots, as well as to estimate changes induced by interventions such as calorie restriction and exercise (21). Owing to CT-induced radiation and the relatively high cost of image analysis with MRI, abdominal adiposity (the VAT and abdominal subcutaneous adipose tissue (ASAT) depot)) is in general characterized using a single cross-sectional image at L2/L3 or L4-L5 level (22). A multi-slice protocol using slices covering the whole abdomen is, however, more accurate when intersubject comparisons are performed $(23,24)$.

In the present study, we want to investigate whether exercise alone or in combination with diet-induced weight loss has a preferential effect in reducing VAT. We used a precise MRI multi-slice technique to determine the size and the changes of the various fat depots. The investigation was a 12-week intervention with three groups of obese subjects randomized to regular exercise alone, diet-induced weight loss, and a combination of exercise and diet-induced weight loss. In the last two groups, we intended to obtain similar weight losses in order to see the possible specific - weight independent - effect of exercise.

\section{Methods}

\section{Subjects}

Seventy-nine obese but otherwise healthy males and females were recruited via advertisements in local newspapers. The subjects were eligible for inclusion if they were aged $18-45$ years, obese $\left(30 \mathrm{~kg} / \mathrm{m}^{2}<\right.$ body mass index (BMI) $<40 \mathrm{~kg} / \mathrm{m}^{2}$ ), physically inactive $(<30 \mathrm{~min} /$ day $)$, and weight stabile for at least three months ( $\pm 2 \mathrm{~kg}$ of current body weight). Exclusion criteria were cardiovascular disease, type 2 diabetes, and pregnancy or orthopedic difficulties causing inability to undertake an exercise program. No subjects received medication that could affect the investigated metabolic markers. Prior to participation, the subjects gave a written informed consent. The study was approved by the local ethics committee in the county of Aarhus and followed the principles outlined in the Declaration of Helsinki.

\section{Study design}

During a 6-month period, the 79 subjects were randomized into the 12-week intervention study consisting of i) exercise only (EXO, $n=25)$, ii) hypocaloric diet (DIO, $n=29$ ), and iii) hypocaloric diet and exercise (DEX, $n=25$ ). Twenty subjects did not complete the study ( 8 women and 12 men; BMI
$35.7 \pm 4 \mathrm{~kg} / \mathrm{m}^{2} ; P=0.2$ as compared with subjects who completed the study). The dropout rate was the highest in the DIO group $(n=10)$ and similar in the EXO $(n=6)$ and DEX groups $(n=4)$. The most frequent reason for subjects in the DIO group leaving the study was dissatisfaction with the group assignment (five subjects).

\section{Diet regime}

Subjects in the DIO and DEX groups were prescribed a liquid very low energy diet (VLED; Nupo, Copenhagen, Denmark) of respectively 600 and $800 \mathrm{kcal} /$ day (proteins $41 \mathrm{~g}$, carbohydrates $29 \mathrm{~g}$, fat $5.6 \mathrm{~g} / 100 \mathrm{~g}$ ) for 8 weeks followed by a weight maintenance diet for 4 weeks. In these two groups, we intended the subjects to obtain similar weight losses in order to see the possible specific - weight independent - effect of exercise. Thus, the subjects in the DEX group were allowed to consume 150-200 kcal more per day as compared with the DIO group, reflecting the estimated extra energy expenditure of $1500 \mathrm{kcal} /$ week during exercise activity. To ensure compliance to the diet, subjects in both groups were allowed to consume ad libitum low-energy vegetables and were followed every second week by clinical staff. In the weight maintenance phase, the subjects consumed a diet with the following energy contents: $55 \%$ from carbohydrates, $15 \%$ from protein, and $<30 \%$ from fat. The daily energy requirement for the subjects during this period was determined by estimating resting energy expenditure multiplied by a factor of 1.5 for subjects in the DIO group and 2.5 in the DEX group. The subjects in the EXO group were advised to maintain an isocaloric diet for the duration of the intervention. Thus, the possible change in the body composition would be due to the differences in the energy expenditure during the exercise activity. Their daily energy expenditure during the intervention was determined by estimating the resting energy expenditure multiplied by a factor of 2.5 . All subjects in the three groups were asked to keep dietary intake records over a 2 week period.

\section{Exercise regime}

The exercise intervention for subjects in the EXO and the DEX group consisted of supervised aerobic exercise three times per week with a duration of 60-75 min per training session, with an estimated energy expenditure of 500-600 kcal per session. The subjects could choose between different modes of exercise; stationary bicycling, jogging on a treadmill or stair stepping. The Karvonen method for exercise intensity was used to target an exercise intensity of $70 \%$ of heart rate reserve: $\mathrm{HR}=\left(\left(\mathrm{HR}_{\max }-\mathrm{HR}_{\text {rest }}\right) \times \%\right.$ Intensity $)+\mathrm{HR}_{\text {rest }}$, where the heart rate maximum was determined during the $\mathrm{VO}_{2}$ max test. The exercise intensity was monitored using heart rate monitors (Polar S810i, Polar Electro 
Oy, Kempele, Finland). The subjects were required to keep records of training sessions during the whole intervention.

\section{Maximal rate of oxygen uptake}

At baseline and after 12 weeks, each subject completed a progressive maximal exercise test using a stationary cycle ergometer (Monark 828, Monark Exercise AB, Vansbro, Sweden) and standard open-circuit spirometry techniques (AMIS 2001, Innovision, Odense, Denmark). All subjects fulfilled two of the following three criteria for maximal aerobic exercise; respiratory exchange ratio $>1.1$, a maximal heart rate within the 15 beats of age-predicted maximal heart rate, and a leveling of $\mathrm{VO}_{2}$ despite increase in Watt.

\section{Anthropometry and metabolic risk factors}

At baseline, at week 8 and after 12 weeks, the body weight was measured to the nearest $0.05 \mathrm{~kg}$ with a calibrated scale. The waist circumference was measured with a flexible measuring tape placed between the costal inferior border and the iliac crest. The hip circumference was measured at the widest point of the hip. Blood pressure was measured on the left arm with the use of an automated blood pressure monitor after the subjects had $5 \mathrm{~min}$ at rest. Blood samples were collected after an overnight fast and at least $24 \mathrm{~h}$ after the subjects had finished the last exercise session and were analyzed at the local department of clinical biochemistry. Cholesterol, triglycerides, and glucose were quantified in plasma. Insulin was analyzed with an ELISA kit assay. The homeostasis model assessment (HOMA) insulin resistance index was calculated using the formula: fasting insulin $(\mathrm{uU} / \mathrm{ml}) \times$ fasting glucose $(\mathrm{mmol} / \mathrm{l}) / 22(25)$.

\section{MRI-protocol}

To quantify the body fat distribution and the effects of the intervention on the regional distribution of adipose tissue (AT) and skeletal muscle (SM), whole body MRI (270 equidistant images) data were obtained at baseline and after 12 weeks with a Philips Gyroscan Achieva 1.5 Tesla MR scanner, (Philips Medical systems, Best, The Netherlands). After a rapid survey scan, a sequence consisting of six stacks each with 45 transversal images were acquired. The sequence was a T1 weighted fast spin echo sequence with slice thickness of $6 \mathrm{~mm}$ and interslice spacing of $1 \mathrm{~mm}$, field of view of $530 \times 530 \mathrm{~mm}^{2}$ and acquisition matrix of $288 \times 202$. The repetition time was $210 \mathrm{~ms}$, echo time $17.5 \mathrm{~ms}$, and the total sequence time was $1109 \mathrm{~min}$.

During acquisition of the images, the subjects rested on the scanner bed in supine position with their arms extended above their heads. To minimize respiratory artifacts a breath hold technique was used at the level of
L5/L4 and 20 images up. To determine the regional effect of the intervention, the whole body was divided into an upper region and a lower region. The upper region: the abdominal adiposity (VAT and ASAT depots) was assessed from the superior position of the femur until no more visceral fat was visible at the level of TH9/TH8 (on average 53 slices). The lower region: GFAT was quantified using 12 slices from the superior position of the femur and $10 \mathrm{~cm}$ down using six slices from the top of patella and $5 \mathrm{~cm}$ up. Intermuscular femoral AT (IMAT) and muscle mass was calculated with six slices from the top of patella and $5 \mathrm{~cm}$ up. The single slice analysis was performed at the L3/L2 level. Fat mass (FM) was calculated as the sum of VAT, ASAT, GFAT, and IMAT. Analysis: To quantify the volume of the respective regions all images were loaded into the Hippo Fat software (26). The program uses a fuzzy c-mean algorithm to segment the image in three tissue classes (air, AT, and non-AT). After this step, external and internal ASAT boundaries were defined by an active contour algorithm. A third contour was computed surrounding the area where VAT was present together with other tissues. VAT itself was assessed by the analysis of the signal in the visceral region by identifying the second peak of the signal histogram. All the images for each subject were manually examined after the automatic analysis in order to correct eventual errors. Analyses using the Hippo fat software have shown high inter- and intra-tester reliability $(27,28)$.

\section{Statistical analysis}

The statistical software packet SPSS (Chicago, IL, USA) was used for all statistical evaluation. Descriptive statistics are presented as means \pm s.D. or as means with confidence interval $(\mathrm{CI})=95 \%$. Group differences at baseline were examined using MANOVA with adjustment for multiple comparisons. The absolute changes and percent changes from baseline to week 12 were calculated for selected variables. MANOVA was used to test the interaction of treatment (EXO, DEX, DIO) and gender (male and female). A linear regression model was used to test the association between selected variables. A non-parametric test was used for variables with a non-normal distribution. The chosen significance level was a two-tailed $P$ value of $<0.05$.

\section{Results}

\section{Baseline characteristics}

The subjects were all obese with a mean BMI of $34.3 \mathrm{~kg} / \mathrm{m}^{2}(103.5 \pm 3 \mathrm{~kg})$ but otherwise healthy. No differences between the three groups in relation to anthropometrics were observed (Table 1).

Of importance, no difference was observed in BMI at baseline between completers and subjects who left prior to the last measurements at week 12 . 


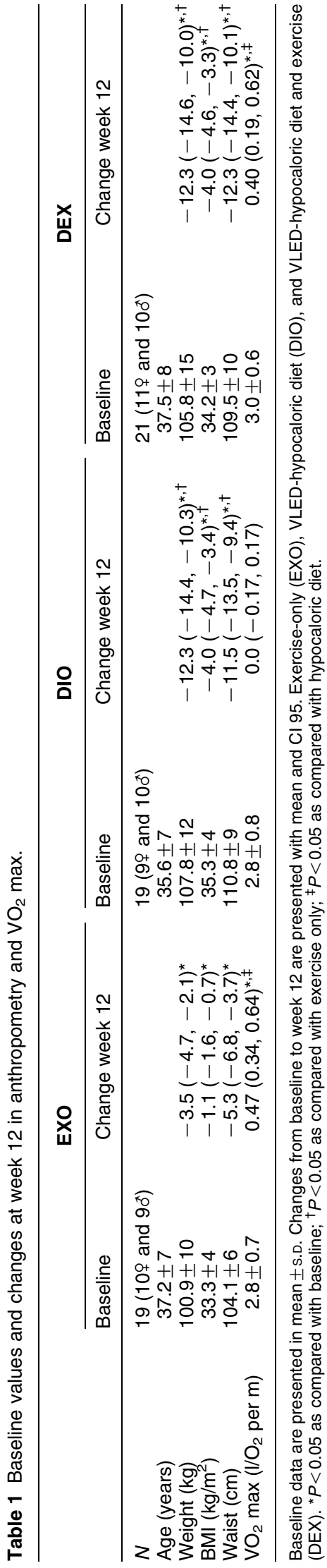

During the 12-week intervention, subjects in the two exercise groups were required to attend 36 supervised exercise sessions with duration of $60-75 \mathrm{~min}$ at an intensity of $70 \%$ of calculated heart rate reserve (HRR) per session. The actual attendance was $96 \%$ with duration of $65 \mathrm{~min}$ and a mean heart rate of 145 beats/min ( $70 \%$ of HRR). The compliance to the VLED in the DIO and DEX groups was $92 \%$ throughout the intervention.

The values for average daily energy intake in the EXO group were comparable before and at the end of the intervention $(2610 \pm 582$ vs $2467 \pm 410 \mathrm{kcal} /$ day; $P=0.6)$.

\section{Body weight, $V_{2} \max$, and fat distribution}

Changes in body weight, fat distribution, and $\mathrm{VO}_{2}$ max after the 12-week intervention are displayed in Fig. 1 and Table 1. Subjects in the EXO group improved their $\mathrm{VO}_{2}$ max by $18 \%(2.8 \pm 0.7$ to $3.4 \pm 0.7 \mathrm{l} / \mathrm{min}$; $P<0.01)$. Body weight was reduced by $3.5 \%$ $(-3.5 \pm 3 \mathrm{~kg} ; P<0.01)$ together with a significant reduction in waist circumference $(-5.3 \mathrm{~cm} ; P<0.01)$ as shown in Table 1 . The relative reduction in all of the anthropometrical parameters was, however; significantly lower as compared with the reductions found in the DIO and DEX groups (Table 1). Weight loss in the DEX and DIO groups were $\sim 11 \%$ after 12 weeks. Reduction of all the other anthropometric parameters in the DEX and DIO groups were also comparable (Table 1; for all comparisons $P>0.05$ ). Subjects in the DEX group increased their $\mathrm{VO}_{2}$ max with $14 \%(P<0.01)$ whereas, no changes in $\mathrm{VO}_{2}$ max were observed in the DIO group.

From the MRI-scanning, it was found that the GFAT, the ASAT, and the VAT in the EXO group were

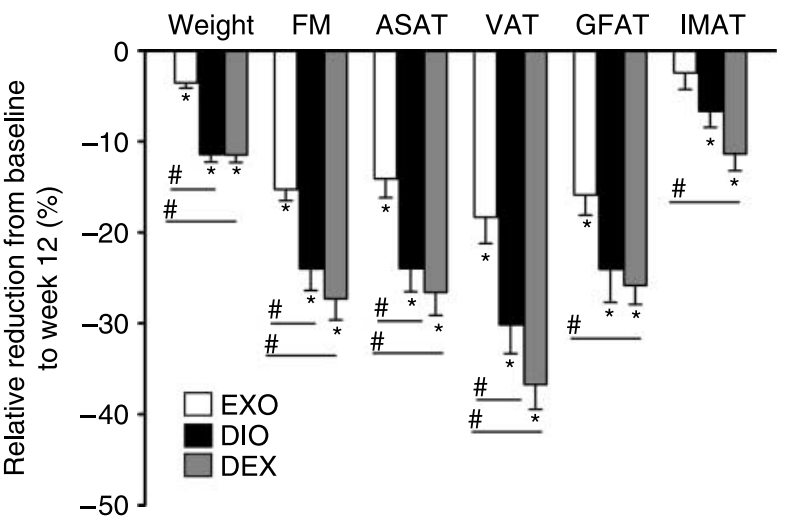

Figure 1 Changes in body weight and body fat distribution. Relative changes in body weight, fat mass (FM, determined as the combination of ASAT + VAT + GFAT + IMAT), subcutaneous abdominal AT (ASAT), visceral abdominal AT (VAT), gluteal femoral AT (GFAT), and in intermuscular adipose tissue (IMAT) in the three groups determined by MRI. Exercise-only (EXO), VLEDhypocaloric diet (DIO), and VLED-hypocaloric diet and exercise (DEX). ${ }^{*} P<0.01$ as compared with baseline, ${ }^{\#} P<0.01$ compared with changes in the EXO group. 
significantly reduced but to a similar degree in the three depots (reduction by $14-18 \% ; P<0.01$, Fig. 1). Moreover, the IMAT was non-significantly reduced by $2 \%$ after 12 weeks of exercise. The reduction in ASAT and VAT was significantly lower in the EXO group as compared with the DIO and DEX groups (Fig. 1). As shown in Fig. 2, the relative reduction of VAT was significantly higher as compared with the relative reduction in FM $(16 \% ; P<0.01)$ in the EXO group. In the DIO group GFAT, ASAT, and VAT were significantly reduced compared with baseline but to a similar degree in all three depots (reduction by $24-30 \%, P<0.01$ ). In the DEX group ASAT, GFAT, and VAT were reduced with 25-37\%; $P<0.01$ as compared with baseline, which were similar to the observed reductions in the DIO group (24-30\%; Fig. 1). As illustrated in Fig. 2, the intervention resulted in a relatively higher loss of VAT as compared with FM both in the DIO group (27\% higher; $P<0.01)$ and in the DEX group (31\% higher; $P<0.01)$. The observed changes in the VAT/FM ratio in the DEX and DIO groups were not significantly different from each other $(P=0.9$, Fig. 2$)$ and these differences were neither different from the $16 \%$ higher reduction of VAT as compared with FM in the EXO group $(P=0.5)$. The IMAT was reduced by $7 \%$ after the diet-induced weight loss $(P<0.01$, Fig. 1$)$ in the DIO group, whereas subjects in the DEX group obtained a reduction of $11 \%(P<0.01$ as compared with baseline but only a non-significant reduction as compared with the DIO group $(P=0.06)$. The muscle mass determined by MRI was reduced with $4-8 \%$ in the DEX and DIO group $(P<0.05)$ and unchanged in the EXO group $(+0.2 \%$; $P=0.6$; data not shown).

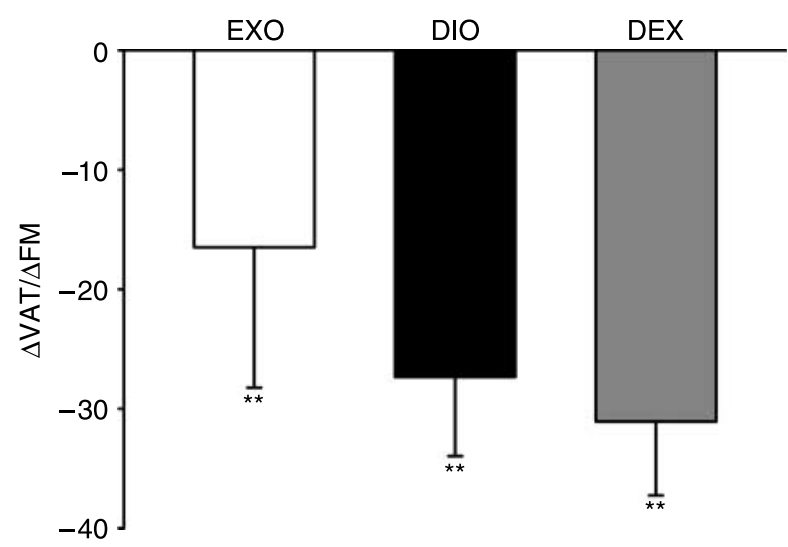

Figure 2 Changes in visceral fat and fat mass. The bar represents $(\Delta \mathrm{FM} \%-\Delta \mathrm{VAT} \% \times 100) / \Delta \mathrm{FM} \%$ in the three groups. Exercise-only (EXO), VLED-hypocaloric diet (DIO), and VLED-hypocaloric diet and exercise (DEX). Fat mass (FM) was calculated as the sum of VAT, ASAT, GFAT, and IMAT. There are no significant differences between the groups (EXO 16\% versus DEX $31 \% ; P=0.5$ ) and DEX $31 \%$ versus DIO $27 \% ; P=0.9) .{ }^{\star \star} P<0.01$ - the relative reduction in VAT as compared with the relative reduction in FM.

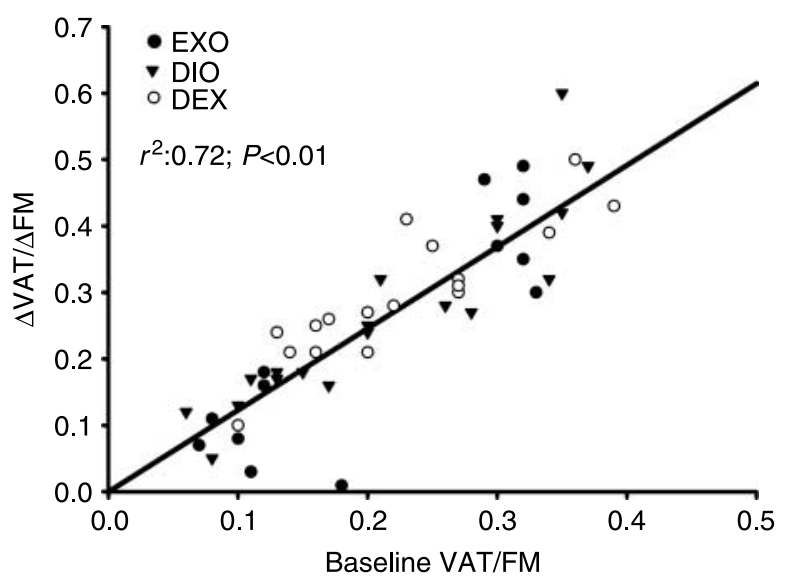

Figure 3 Changes of visceral fat relative to changes in fat mass. Changes of VAT relative to changes in FM ( $\triangle \mathrm{VAT} / \Delta \mathrm{FM}-y$-axis) versus the initial VAT/FM ratio ( $x$-axis) in the three groups;

, exercise-only (EXO), $\boldsymbol{\nabla}$, VLED-hypocaloric diet (DIO), and $\bigcirc$, VLED-hypocaloric diet and exercise (DEX). Fat mass (FM) was calculated as the sum of VAT, ASAT, GFAT, and IMAT from MRI determinations.

\section{The relationship between VAT and FM}

The relationship between the initial ratio of VAT and FM and the ratio between changes in VAT and FM in the three groups is illustrated in Fig. 3. It was found that the initial VAT/FM ratio was similar between the three groups (data not shown) and we observed a linear relationship $\left(R^{2}=0.72 ; P<0.01\right)$ between changes in VAT and changes in FM ( $y$-axis). As shown, no type of intervention deviated from the regression line, reflecting that changes in VAT were primarily determined by changes in FM and the initial VAT/FM ratio and independent of the intervention.

\section{Influence of gender}

As expected, males had lower ASAT volume $\left(7897 \pm 2788\right.$ vs $\left.11523 \pm 2307 \mathrm{~cm}^{3} ; \quad P<0.001\right)$, lower GFAT volume $\left(1975 \pm 624\right.$ vs $3680 \pm 821 \mathrm{~cm}^{3}$; $P<0.001)$, and significantly higher VAT volume $\left(3846 \pm 1072\right.$ vs $\left.2268 \pm 938 \mathrm{~cm}^{3} ; \quad P<0.001\right)$ as compared with females (data not shown). The VAT/ ASAT ratio $(0.5 \pm 15$ vs $0.2 \pm 0.6 ; P<0.001)$ and VAT/GFAT ratio $(2.0 \pm 0.7$ vs $0.7 \pm 0.3 ; P<0.001)$ were higher in males as compared with females. Concerning the relative changes in the different AT depots during the intervention, there were no differences between females and males - EXO group: reduction in VAT (females $16 \pm 9 \%$ versus males $20 \pm 6 \% ; P=0.4$ ); GFAT (females 15 \pm 5\% versus males $16 \pm 9 \% ; P=0.8$ ); ASAT (females $14 \pm 5 \%$ versus males $16 \pm 10 \% ; P=0.7$ ) - DIO group: VAT (females $28 \pm 10 \%$ versus males $31 \pm$ $16 \%$; $P=0.6$ ); GFAT (females $24 \pm 4 \%$ versus males 
$24 \pm 12 \% ; P=0.9$ ); ASAT (females $22 \pm 5 \%$ versus males $25 \pm 10 ; P=0.5)$, and in the DEX group: VAT (females $35 \pm 12 \%$ versus males $38 \pm 13 \%$; $P=0.7$ ); GFAT (females $24 \pm 8 \%$ versus males $27 \pm 12 \%$; $P=0.6$ ); ASAT (females $25 \pm 9 \%$ versus males $28 \pm 10 \% ; P=0.4$; data not shown). No differences were observed between males and females in the three groups in relation to relative changes in anthropometry (data not shown).

\section{MRI multi-slice versus MRI single-slice}

To investigate the possible discrepancy using a MRI multi-slice protocol versus a single-slice protocol at $\mathrm{L} 3 / \mathrm{L} 2$ level in relation to the relative reduction in VAT and ASAT, we compared the two MRI protocols. In all the three groups, the relative reduction in VAT was comparable between the two MRI-protocols; EXO (single-slice $14 \%$ versus multi-slice $17 \%$; intraclass correlation (ICC) 0.55 ; $P<0.05$ ), DIO group (singleslice 33\% versus multi-slice 30\%, ICC $0.52 ; P<0.01$ ), and DEX group (single-slice $34 \%$ versus multi-slice 37\%; ICC 0.55; P<0.05). Similarly comparable results between the two MRI protocols were observed in relation to the relative ASAT reduction; EXO group (single slice $14 \%$ versus multi-slice $14 \%$; ICC 0.58 ; $P<0.01$ ), DIO group (single slice $27 \%$ versus multi-slice $25 \%$; ICC $0.64 ; P<0.01$ ), and DEX group (single slice $27 \%$ versus multi-slice $28 \%$; ICC $0.8 ; P<0.01$; data not shown).

\section{Association between $\mathrm{VO}_{2} \max$ increment and the decrease in VAT}

To investigate if the magnitude of the $\mathrm{VO}_{2}$ max was associated with the reduction of VAT, the subjects in the two exercise groups (EXO and DEX) were divided into three groups (tertiles) in relation to their increment of $\mathrm{VO}_{2}$ max at week 12. Comparing subjects in the lowest quartile of $\mathrm{VO}_{2}$ max (increase in $\mathrm{VO}_{2}$ max with 5\%) with subjects in the highest quartile (increase in $\mathrm{VO}_{2}$ max with $25 \%$ ), we found a comparable reduction of VAT in the two groups $(28 \%$ vs $32 \% ; P=0.4$; data not shown).

\section{The metabolic profile}

The changes in the metabolic profile after 12 weeks in the three groups are illustrated in Table 2. Total cholesterol and blood pressure were significantly reduced in all three groups, whereas only in the DIO and DEX groups, a significant decrease in triglycerides, glucose, and insulin was observed. A trend toward a significant decrease in insulin $(P=0.06)$ and HOMA $(P=0.09)$ was observed in the EXO group. Of importance, high density lipoprotein (HDL)-cholesterol was significantly increased in the DEX group $(P<0.05)$.

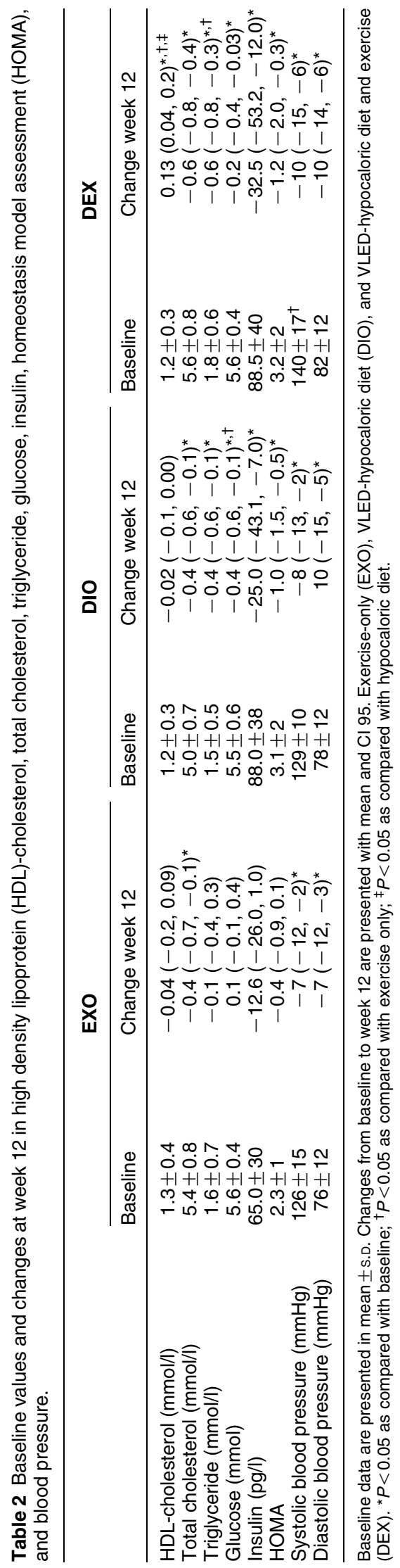

www.eje-online.org 


\section{Discussion}

The primary finding in the present study was that the carefully matched weight loss in the two groups (DIO $12.3 \mathrm{~kg}$ versus DEX $12.3 \mathrm{~kg}$ ) displayed that exercise has no weight independent effect on the VAT depot. Instead, changes in the VAT depot seems to be closely associated with changes in the FM and related to the initial VAT/FM ratio and independent of the intervention program. This interpretation is also in agreement with the results obtained in the EXO group where the combined effect of exercise - effect through exerciseinduced weight loss (negative energy balance) and the effect of exercise per se - on fat distribution was examined.

The health complications of obesity are particularly related to the amount of VAT. Accordingly, it is of great clinical interest whether preferential reduction of VAT can be induced. This might be an easier way of obtaining health benefits for obese subjects, since large weight loss and weight loss maintenance are difficult to obtain $(6,7)$. Exercise has by some groups been suggested to have a preferential effect in reducing the VAT depot (12-17). It has been shown that various hormones (e.g. corticosteroids) and drugs (e.g. PPAR $\gamma$ agonists) may have specific effects on fat distribution $(8,29)$. Exercise might affect fat distribution by different mechanisms. It is known that exercise increases the level of catecholamines (30) and catecholamines have effects on adipocyte lipolysis through $\beta$-adrenergic receptors (stimulation) and $\alpha 2$-adrenergic receptors (inhibition) (31). Previously, we have shown that catecholamines are more lipolytically active in visceral adipocytes as compared with subcutaneous adipocytes (32) which may result in more pronounced reduction of VAT induced by exercise. Another mechanism for a preferential reduction of VAT induced by exercise might be through central effects of exercise on for example, the hypothalamic-pituitary-adrenal (HPA) axis that may reduce the VAT depot through reduced cortisol turnover (33). However, since we found no preferential effect of exercise on VAT, increased physical activity as obtained in the present study was not able to activate these suggested biological pathways.

The observation that the loss of VAT was proportional to the loss of FM and related to the initial ratio of VAT and FM is in accordance with the suggestions in a recent review (20) that an allometric relationship exists between changes in VAT and FM implying that subjects undergoing obesity treatment will reduce their VAT by about 30\% more as compared with the FM, independently of type of weight loss (exercise, diet, surgery). Thus, the suggestion of a preferential reduction of the VAT depot may often be due to misinterpretation of the weight loss results. For example, the moderate exercise programs inducing preferential losses of VAT shown in the studies by Thomas et al. (16) and Mourier et al. (15) were not compared with diet restriction programs, and the more prominent reduction in VAT adding exercise to a restricted diet compared with a diet restriction alone found by Okura et al. (14) did not take into account that changes in VAT and FM are parallel and determined by the initial VAT/FM ratio. In addition, the observation that exercise was essential for a decrease in the VAT depot in type 2 diabetics (13) was made on the absolute changes in the VAT depot and not on the relative changes and again not taking into account that changes in VAT and FM are parallel and determined by the initial VAT/FM.

Our findings in the present study are in agreement with Redman et al. (34) who found that subjects who lost $\sim 10 \%$ of their initial body weight after calorie restriction or combined calorie restriction and exercise achieved a reduction in the VAT depot of $\sim 27 \%$ with no differences between the two groups. Since our sample size is larger than the study by Redman et al. $(n=12$ in each group) the observed reduction in the VAT depot in the two studies may not be due to any type two error. We therefore suggest that an allometric relationship exists between VAT and FM which determines the reduction of the VAT depot and that the reduction of the VAT depot will be $20-30 \%$ higher than the reduction of FM independent of the type of intervention. Adding exercise to a weight reducing diet results in greater improvements in the metabolic abnormalities compared with a weight reducing diet alone $(35,36)$. In the present study, we found only an additive effect of exercise on HDLcholesterol when comparing the results in the two hypocaloric groups. This may be due to the fast and massive impact of a VLED on metabolic parameters with near-normalization of insulin, glucose, and lipid-levels (37), which could have obscured a more gentle effect of exercise. In addition to the increase in HDL-cholesterol, the observed increase in $\mathrm{VO}_{2}$ max in the DEX group is of importance. Increased $\mathrm{VO}_{2}$ max is associated with several physiological adaptations in SM, e.g., improved mitochondrial function and enhanced muscle oxidative capacity, whereas a low level of aerobic fitness is a predictor of cardiovascular diseases (38). The effect of exercise on body composition and metabolic parameters is well described (39) and is generally confirmed in this study. Subjects in the EXO group reduced their body weight with $3.5 \%$ and their VAT and ASAT with $17 \%$ and $14 \%$ respectively. The alteration in body composition was accompanied with significant reductions in blood pressure, total cholesterol, and a trend toward a significant decrease in HOMA $(P=0.09)$. In relation to the IMAT, we found a trend toward a higher relative reduction in the DEX group after 12 weeks as compared with the DIO group $(P=0.06)$. IMAT is associated with impaired insulin sensitivity and increased cholesterol levels (40).

The effect of diet-induced weight loss and exercise on metabolic parameters and changes in the regional fat distribution seem to be independent of gender, since the changes observed in females and males were comparable. In this study, we used a multi-slice protocol to 
cover VAT and ASAT (on average 53 slices) and to cover GFAT (on average 20 slices). When comparing the ratio between the relative reduction of VAT and ASAT obtained using a multi-slice protocol and single-slice protocol, we found relatively consistent results. This observation implies that the discrepancy in literature, of whether exercise has preferential effect on VAT or not is not related to the MRI protocol (multi-slice protocol versus single-slice protocol) but may be more down to the interpretation of the results.

A major strength of the present study was the use of a multi-slice protocol to cover VAT and ASAT depots, use of a randomized design including males and females, a carefully monitored diet of all subjects, and the supervised exercise session in the EXO and DEX groups. There are some limitations to the study. The relative high dropout rate in the DIO group (10 subjects) could introduce a selection bias but since the weight loss in the DIO and the DEX groups was similar as planned we do not think this dropout rate had any effect on our results. As mentioned, the weight loss independent effects of exercise may have been somehow obscured in the present study by the pronounced diet-induced weight loss and more effects of exercise on metabolic parameters would presumably have been observed with more moderate weight loss. However, the conclusion concerning no specific effect of exercise on fat distribution is valid since we have also included an exercise-alone group in the study where weight loss was allowed and the reduction in VAT in this group was as predicted from the allometric relationship.

In summary, we found that weight loss induced by exercise has no preferential effect in reducing the VAT depot as compared with diet-induced weight loss. Furthermore, changes in the VAT depot are paralleled with the change in FM and determined by the initial VAT to FM ratio independent of the weight loss program applied. In relation to the observed changes in the metabolic parameters, our observations suggest that the independent and additive effect of exercise cannot be related to a specific reduction of the VAT depot induced by exercise. Finally, it was found that the single slice MRI is as good as the multi-slice MRI to determine changes in fat distribution.

\section{Declaration of interest}

The authors declare that there is no conflict of interest that could be perceived as prejudicing the impartiality of the research reported.

\section{Funding}

The study was supported by Aarhus University, Novo Nordic Foundation, The Danish Diabetic Association, and the Society of Physiotherapists in Denmark.

\section{Acknowledgements}

We thank Lenette Pedersen and Pia Hornbek for their skillful technical assistance.

\section{References}

1 Despres JP. Is visceral obesity the cause of the metabolic syndrome? Annals of Medicine 200638 52-63.

2 Flegal KM, Graubard BI, Williamson DF \& Gail MH. Cause-specific excess deaths associated with underweight, overweight, and obesity. Journal of the American Medical Association 2007298 2028-2037.

3 Tanko LB, Bagger YZ, Alexandersen P, Larsen PJ \& Christiansen C. Central and peripheral fat mass have contrasting effect on the progression of aortic calcification in postmenopausal women. European Heart Journal 200324 1531-1537.

4 Tanko LB, Bruun JM, Alexandersen P, Bagger YZ, Richelsen B, Christiansen C \& Larsen PJ. Novel associations between bioavailable estradiol and adipokines in elderly women with different phenotypes of obesity: implications for atherogenesis. Circulation $20041102246-2252$.

5 Yusuf S, Hawken S, Ounpuu S, Bautista L, Franzosi MG, Commerford P, Lang CC, Rumboldt Z, Onen CL, Lisheng L, Tanomsup S, Wangai P Jr, Razak F, Sharma AM, Anand SS, INTERHEART Study Investigators. Obesity and the risk of myocardial infarction in 27000 participants from 52 countries: a case-control study. Lancet 2005366 1640-1649.

6 Christiansen T, Bruun JM, Madsen EL \& Richelsen B. Weight loss maintenance in severely obese adults after an intensive lifestyle intervention: 2- to 4-year follow-up. Obesity $2007 \mathbf{1 5}$ 413-420.

7 Wadden TA. Treatment of obesity by moderate and severe caloric restriction. Results of clinical research trials. Annals of Internal Medicine 1993119 688-693.

8 Akazawa S, Sun F, Ito M, Kawasaki E \& Eguchi K. Efficacy of troglitazone on body fat distribution in type 2 diabetes. Diabetes Care 200023 1067-1071.

9 Malis C, Rasmussen EL, Poulsen P, Petersen I, Christensen K \& Beck-Nielsen $\mathrm{H}$. Total and regional fat distribution is strongly influenced by genetic factors in young and elderly twins. Obesity Research 200513 2139-2145.

10 Carey DG, Nguyen TV, Campbell LV, Chisholm DJ \& Kelly P. Genetic influences on central abdominal fat: a twin study. International Journal of Obesity and Related Metabolic Disorders 199620 $722-726$.

11 Ross R, Janssen I, Dawson J, Kungl AM, Kuk JL, Wong SL, Nguyen-Duy TB, Lee S, Kilpatrick K \& Hudson R. Exercise-induced reduction in obesity and insulin resistance in women: a randomized controlled trial. Obesity Research 200412 789-798.

12 Lee S, Kuk JL, Davidson LE, Hudson R, Kilpatrick K, Graham TE \& Ross R. Exercise without weight loss is an effective strategy for obesity reduction in obese individuals with and without Type 2 diabetes. Journal of Applied Physiology 200599 $1220-1225$.

13 Giannopoulou I, Ploutz-Snyder LL, Carhart R, Weinstock RS, Fernhall B, Goulopoulou S \& Kanaley JY. Exercise is required for visceral fat loss in postmenopausal women with type 2 diabetes. Journal of Clinical Endocrinology and Metabolism 200590 1511-1518.

14 Okura T, Nakata Y, Lee DJ, Ohkawara K \& Tanaka K. Effects of aerobic exercise and obesity phenotype on abdominal fat reduction in response to weight loss. International Journal of Obesity 200529 1259-1266.

15 Mourier A, Gautier JF, De Kerviler E, Bigard AX, Villette JM, Garnier JP, Duvallet A, Guezennec CY \& Cathelineau G. Mobilization of visceral adipose tissue related to the improvement in insulin sensitivity in response to physical training in NIDDM. Effects of branched-chain amino acid supplements. Diabetes Care 199720 385-391.

16 Thomas EL, Brynes AE, McCarthy J, Goldstone AP, Hajnal JV, Saeed N, Frost G \& Bell JD. Preferential loss of visceral fat following aerobic exercise, measured by magnetic resonance imaging. Lipids 200035 769-776. 
17 Lynch NA, Nicklas BJ, Berman DM, Dennis KE \& Goldberg AP. Reductions in visceral fat during weight loss and walking are associated with improvements in $\mathrm{VO}(2 \mathrm{max})$. Journal of Applied Physiology 200190 99-104.

18 Lakka TA \& Laaksonen DE. Physical activity in prevention and treatment of the metabolic syndrome. Applied Physiology, Nutrition, and Metabolism 200732 76-88.

19 Chaston TB \& Dixon JB. Factors associated with percent change in visceral versus subcutaneous abdominal fat during weight loss: findings from a systematic review. International Journal of Obesity 200832 619-628.

20 Hallgreen CE \& Hall KD. Allometric relationship between changes of visceral fat and total fat mass. International Journal of Obesity $200832845-852$.

21 Goodpaster BH. Measuring body fat distribution and content in humans. Current Opinion in Clinical Nutrition and Metabolic Care 20025 481-487.

22 Shen W, Wang Z, Punyanita M, Lei J, Sinav A, Kral JG, Imielinska C, Ross R \& Heymsfield SB. Adipose tissue quantification by imaging methods: a proposed classification. Obesity Research 200311 5-16.

23 Kanaley JA, Giannopoulou I \& Ploutz-Snyder LL. Regional differences in abdominal fat loss. International Journal of Obesity 200731 147-152.

24 Thomas EL \& Bell JD. Influence of undersampling on magnetic resonance imaging measurements of intra-abdominal adipose tissue. International Journal of Obesity and Related Metabolic Disorders 200327 211-218.

25 Matthews DR, Hosker JP, Rudenski AS, Naylor BA, Treacher DF \& Turner RC. Homeostasis model assessment: insulin resistance and beta-cell function from fasting plasma glucose and insulin concentrations in man. Diabetologia 198528 412-419.

26 Positano V, Gastaldelli A, Sironi AM, Santarelli MF, Lombardi M \& Landini L. An accurate and robust method for unsupervised assessment of abdominal fat by MRI. Journal of Magnetic Resonance Imaging $200420684-689$.

27 Arif H, Racette SB, Villareal DT, Holloszy JO \& Weiss EP. Comparison of methods for assessing abdominal adipose tissue from magnetic resonance images. Obesity 200715 2240-2244.

28 Bonekamp S, Ghosh P, Crawford S, Solga SF, Horska A, Brancati FL, Diehl AM, Smith S \& Clark JM. Quantitative comparison and evaluation of software packages for assessment of abdominal adipose tissue distribution by magnetic resonance imaging. International Journal of Obesity 200832 100-111.

29 Mattsson C \& Olsson T. Estrogens and glucocorticoid hormones in adipose tissue metabolism. Current Medicinal Chemistry 200714 2918-2924.
30 Martin WH III. Effects of acute and chronic exercise on fat metabolism. Exercise and Sport Sciences Reviews $1996 \mathbf{2 4}$ 203-231.

31 Richelsen B. Increased alpha 2- but similar beta-adrenergic receptor activities in subcutaneous gluteal adipocytes from females compared with males. European Journal of Clinical Investigation 198616 302-309.

32 Richelsen B, Pedersen SB, Moller-Pedersen T \& Bak JF. Regional differences in triglyceride breakdown in human adipose tissue: effects of catecholamines, insulin, and prostaglandin $\mathrm{E}_{2}$. Metabolism 199140 990-996.

33 Bjorntorp P. Do stress reactions cause abdominal obesity and comorbidities? Obesity Reviews 20012 73-86.

34 Redman LM, Heilbronn LK, Martin CK, Alfonso A, Smith SR \& Ravussin E. Effect of calorie restriction with or without exercise on body composition and fat distribution. Journal of Clinical Endocrinology and Metabolism 200792 865-872.

35 Stefanick ML, Mackey S, Sheehan M, Ellsworth N, Haskell WL \& Wood PD. Effects of diet and exercise in men and postmenopausal women with low levels of HDL cholesterol and high levels of LDL cholesterol. New England Journal of Medicine 1998339 $12-20$.

36 Wood PD, Stefanick ML, Williams PT \& Haskell WL. The effects on plasma lipoproteins of a prudent weight-reducing diet, with or without exercise, in overweight men and women. New England Journal of Medicine 1991325 461-466.

37 Henry RR, Wiest-Kent TA, Scheaffer L, Kolterman OG \& Olefsky JM. Metabolic consequences of very-low-calorie diet therapy in obese non-insulin-dependent diabetic and nondiabetic subjects. Diabetes $198635155-164$.

$38 \mathrm{Hu}$ FB, Willett WC, Li T, Stampfer MJ, Colditz GA \& Manson JE. Adiposity as compared with physical activity in predicting mortality among women. New England Journal of Medicine 2004 $3512694-2703$.

39 Shaw K, Gennat H, O'Rourke P \& Del Mar C. Exercise for overweight or obesity. Cochrane Database of Systematic Reviews, $20061-7$.

40 Yim JE, Heshka S, Albu J, Heymsfield S, Kuznia P, Harris T \& Gallagher D. Intermuscular adipose tissue rivals visceral adipose tissue in independent associations with cardiovascular risk. International Journal of Obesity 200731 1400-1405.

Received 10 February 2009

Accepted 11 February 2009 